\title{
Optimized ZRP for MANETs and its Applications
}

\author{
M.N. SreeRangaRaju \\ Department of Telecommunication Engg \\ Bangalore Institute of Technology \\ Bangalore, India \\ Email: mnsrr@rediffmail.com
}

\author{
Dr. Jitendranath Mungara \\ CMR Institute of Tech \& Mgt \\ Bangalore, India \\ Email: jmungara@yahoo.com
}

\begin{abstract}
In this paper, we unified two new algorithms, i.e., to selectively Bordercast the control packets in order to reduce the network load by limiting the number of control packets when ZRP searches for a new route. The second algorithm is proposed to optimize the performance of Query control mechanisms for the Zone Routing Protocol (ZRP) for mobile ad hoc networks for communication on an urban terrain. ZRP proactively maintains routing information for a local neighbourhood (routing zone), while reactively acquiring routes to destinations beyond the routing zone. This hybrid routing approach can be more efficient than traditional Zone Routing Protocol (ZRP) framework. However, without proper query control techniques, the ZRP cannot provide the expected reduction in the control traffic. Our proposed algorithm enhances the structure of the routing zone to provide enhanced detection and prevention of overlapping queries. These techniques can be applied to single or multiple channel mobile adhoc networks to improve both the delay and control traffic performance of ZRP. Our new algorithm allows ZRP to provide routes to all accessible network nodes, with less control traffic than purely proactive link state or purely reactive route discovery, and with less delay than conventional flood searching.
\end{abstract}

To further improve the efficiency of Enhanced ZRP, our future work shall propose a new algorithm to overcome the generation of periodic updates in the routing table to reduce the control traffic. Thus by the implementation of our proposed algorithm the time taken to receive the first packet will be minimized, which results in better throughput.

The performance evaluation via simulations using a well known simulator QualNet version 5.0 shows that the new approach has better performance than the traditional ZRP.

\section{KEYWORDS}

Adhoc network, Bordercast, hybrid routing, Proactive routing, query control, reactive routing, routing protocol, routing zone and zone routing protocol $(Z R P)$.

\section{INTRODUCTION}

Manet is a collection of wireless nodes that can dynamically form a network to exchange information without using any pre-existing fixed network infrastructure with rapid configuration of wireless connections on-the-fly [1]. Due to rapidly changing connectivity, network partition, higher error rates, collision interference, bandwidth and power constrain together pose new problems in network control-particularly in the design of higher level protocol such as routing and in implementing applications with quality of service requirements. Routing in Manets is one of the primary functions that, each node must achieve network functions at any point of time. Routing enables connections between nodes those are not within each other's vicinity. To serve this purpose, various proactive, reactive and hybrid routing protocols are been proposed.

In proactive routing protocols viz., destination-sequenced distance vector (DSDV) [2] and optimized link state routing protocol (OLSR) [3], routes between source and destination are maintained regardless of the data traffic. Such a strategy avoids the need of finding routes for 
each message and is especially efficient when the nodes are relatively stationary and traffic is relatively heavy. Unlike proactive routing protocols, reactive routing protocols [4] viz., dynamic source routing (DSR) and ad hoc on demand distance vector (AODV), find route only when node attempts to send a message. It avoids the need of frequent link and route updates therefore substantially reduces energy consumption [5] when the traffic load is light or the network mobility is high. Hybrid routing protocol utilizes both reactive and proactive protocol mechanisms.

The rest of the paper is organized as follows: section- ii explains overview of DSR, AODV and ZRP for Manets. Section iii gives a detailed description of our proposed work such that we can have better efficiency. Section iv presents simulation based results, evaluation and performance comparison graphs of our previous work. Finally, conclusion and future work are presented in section $\mathrm{v}$.

\section{Overview of ZRP}

\subsection{Zone Routing Protocol}

ZRP was proposed to reduce the control overhead of proactive routing protocols and decrease the latency caused by routing discover in reactive routing protocols. ZRP defines a zone around each node consisting of its k-neighbourhood (e. g. $\mathrm{k}=3$ ). In $\mathrm{ZRP}$, the distance and a node, all nodes within -hop distance from node belongs to the routing zone of node. ZRP is formed by two sub-protocols, a proactive routing protocol: Intra-zone Routing Protocol (IARP) [9] is used inside routing zones and a reactive routing protocol: Inter-zone Routing Protocol (IERP) [10] is used between routing zones, respectively. A route to a destination within the local zone can be established from the proactively cached routing table of the source by IARP, therefore, if the source and destination is in the same zone, the packet can be delivered immediately. Most of the existing proactive routing algorithms can be used as the IARP for ZRP. For routes beyond the local zone, route discovery happens reactively. The source node sends a route requests to its border nodes, containing its own address, the destination address and a unique sequence number. Border nodes are nodes which are exactly the maximum number of hops to the defined local zone away from the source. The border nodes check their local zone for the destination. If the requested node is not a member of this local zone, the node adds its own address to the route request packet and forwards the packet to its border nodes. If the destination is a member of the local zone of the node, it sends a route reply on the reverse path back to the source. The source node uses the path saved in the route reply packet to send data packets to the destination.

\section{Related Work}

This section reviews the some of literature pertaining to routing in wireless ad hoc networks. In [1], the work presents query controlled scheme to provide enhanced detection and prevention of overlapping queries. The work done in [2] presents a selective bordercasting zone routing protocol to reduce the network load by limiting the number of control packets when the protocol searches for new route. The work presented in [3], is a protocol called multipath distance vector zone routing protocol for mobile ad-hoc networks, which uses a topological map of the zone centered on a node to guarantee loop freedom and alternative paths in the case of route failure and disjoint paths. In [4] an algorithm is proposed to provide improved quality of service such as low end to end delay and high throughput via hybrid routing protocol ZRP. In paper [5] independent zone routing is proposed, which allows adaptive and distributed configuration for the optimal size of the each node's routing zone on the per node basis. $\operatorname{In}[6]$, it has given review about the current routing protocols in Ad-hoc networks. 


\section{Proposed Work}

Basic idea of ZRP is based on the idea that querying can be done more efficiently than flooding, by directing route requests to target peripheral nodes. Since neighbouring routing zones heavily overlap, each node may forward same query messages multiple times. Thus leading to increase in the number of control packets. Also there are chances that these query messages may be forwarded again inward instead of moving towards the destination. This unnecessarily adds the delay in the system.

\subsection{Detection of Query PacketId}

To reduce this delay we can go for query control mechanisms. Query detection uses query source node's Id and query Id pair. Below Fig. 1 depicts query detection scheme. Where ' $S$ ' is the source node and destination node ' $\mathrm{D}$ ' is not in S's local zone. Hence S Bordercasts the query message packet. Nodes J, K, I detects the query packet through QID Method-1 and node $\mathrm{H}$ detects it through evesdropping i.e QID Method-2.

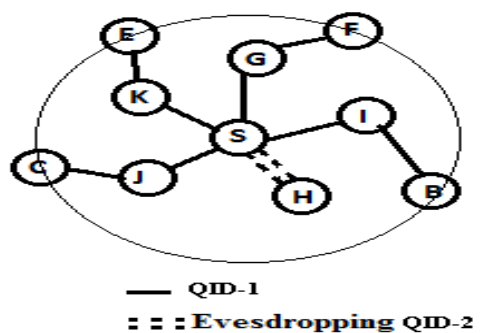

\subsection{Premature Node Termination}

With the knowledge of the QD obtained a procedure has to be followed in which covered nodes can be pruned by the Bordercast tree. This procedure is known as premature node termination. By referring to below Fig. 2, we can see that node ' $S$ ' Bordercasts the query message packet to its peripheral nodes. Node 'I' receives same copy of query message packet through Bordercast tree by the other node as it may be interior member of other routing zone. Now 'I' searches for the interior zone member of other node, and uses QD to detect is it necessary to relay that query message packet or no. 'I' identifies that nodes are been covered, it reconstructs the Bordercast tree. Prunes covered nodes from other node' Bordercast tree and deletes the query message received by other node using the criterion called premature node termination.

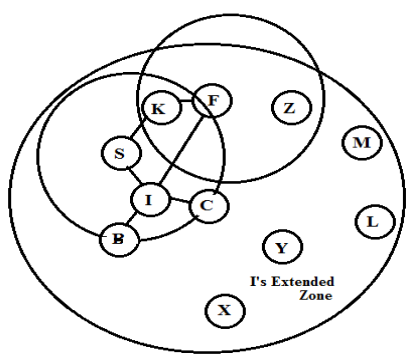

Fig. 2: Premature Node Termination 


\subsection{Random Query Analysing and Processing Delay}

Bordercasting node initiates bordercasting to its peripheral nodes. We can observe from the below Fig. 3 that bordercasting node ' $S$ ' Bordercasts the query message packet to peripheral node ' $F$ '. This node ' $F$ ' will border cast the query message packet to ' $X$ ' and ' $Y$ '. ' $X$ ' and ' $\mathrm{Y}$ ' receive query at the same time. If both of them Bordercasts the query then again there are chances that same node may relay the same query multiple times. Unless and until $\mathrm{X}$ and $\mathrm{Y}$ check for the QD information they both will not be knowing that the query Bordercasted by them is redundant.

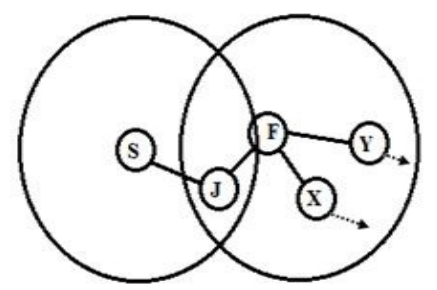

Fig. 3: Random Query Analyzing and Processing Delay

\subsection{Selective Bordercasting Approach:}

This approach is specially proposing for the minimization of control overhead packets for the ZRP protocol, which leads to congestion and delay in route acquisition The selective bordercasting mechanism use same IARP protocol as in ZRP, but use a new mechanism in IERP for interzone routing because the bordercasting procedure starts with IERP protocol.

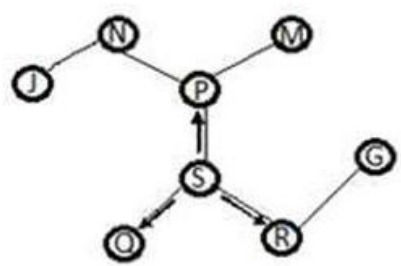

Fig. 4: IARP packet broadcasting.

The operation of the IARP is shown in Fig. 4. The source node $\mathrm{S}$ generates the IARP packet and broadcasts in its local routing zone, by using neighbor's information, the central node updates its routing table. When the destination is outside the routing zone, the interzone operation initializes. The IERP is slight different from IERP in original ZRP, to understand the mechanism of new IERP, consider the Fig. 5, which the route to the destination in unknown to the source. Then the IERP request packet is generated. The information in the new IERP packet is: $\mathrm{SN}=\mathrm{S}, \mathrm{DN}=\mathrm{D}$, and border-cast hop value $(\mathrm{BH})=1$ which is shown in Fig. 5(a) and Fig. 5(b). The generated IERP packet is sent to all border nodes which lie on the zone border, which have same zone radius. 


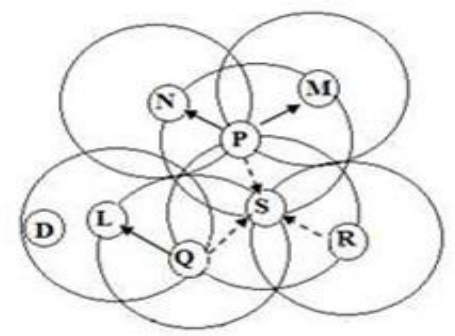

Fig. 5: IERP Packet transmission.

\begin{tabular}{|c|c|c|c|c|}
\hline DN & SN & $\begin{array}{c}\text { Intermediate } \\
\text { Node }\end{array}$ & $\begin{array}{c}\text { Packet } \\
\text { Type }\end{array}$ & BH \\
\hline
\end{tabular}

Fig. 5(a): IERP Packet format.

\begin{tabular}{|c|c|c|c|c|}
\hline DN & SN & $\begin{array}{c}\text { Intermediate } \\
\text { Node }\end{array}$ & $\begin{array}{c}\text { Packet } \\
\text { Type }\end{array}$ & BH \\
\hline D & S & Null & REQ & 1 \\
\hline
\end{tabular}

Fig. 5(b): IERP Request packet format of Fig. 5.

The nodes in the zone boundary which receives the IERP request packet with increase in the $\mathrm{BH}$ value by 1 . Then the node checks the destination in its routing table, if the destination address doesn't found in its routing table, then it should continue with the border casting procedure. When the nodes find the destination in its routing zone or itself a destination then the node will replays with IERP Replay packet as shown in the Fig. 6 and reply packet is shown in Fig. 6(a).

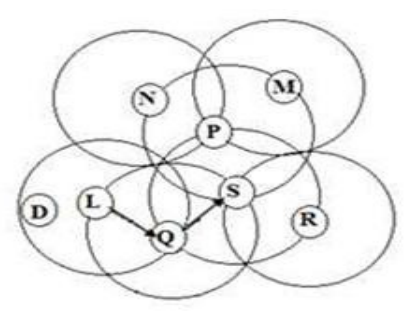

Fig. 6: IERP Reply Transmission

\begin{tabular}{|c|c|c|c|c|}
\hline DN & SN & Intermediate BN & Packet Type & BH \\
\hline D & S & L, Q & REP & 2 \\
\hline
\end{tabular}

Fig. 6(a): IERP reply packet format of Fig-6

In this Selective bordercasting approach the IERP Request packet's must save discovered routing information in a buffer for awhile and if there are requests for the same destination which the IERP REQ's are sent by nodes in the previous search. For example, when a destination moves inside the routing zone of Q, as shown in the Fig. 7, the previous recorded route information stored in the node $\mathrm{Q}$ and $\mathrm{L}$, the node $\mathrm{Q}$ will generate the Reply packet to source $\mathrm{S}$. 


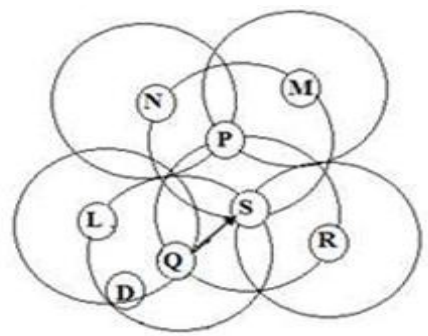

Fig. 7: a case when the destination node moves inside the zone.

In the case of destination which moves outside the zone of $\mathrm{L}$ as shown in the Fig. 8, it has no route to destination which is required for the query source, and then a new search is started from the node $\mathrm{L}$.

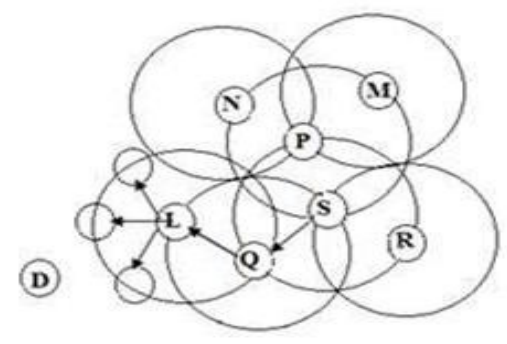

Fig. 8: A case when DN moves outside the zone

Thus the number of IERP Route Request packets is reduced, because the route reconfiguration is started from the destination failure reporting node instead of beginning from the source. Hence this reduces control overhead packets and end-to-end delay time, which results in higher throughput.

\section{Simulation Results}

In this section the methodology is presented .This methodology is used in order to isolate the impact on network performance. We used a well known network simulator QualNet version 5.0 .

\subsection{Simulation Platform, Models and Attributes}

The image of the network as it appears in QualNet version 5.0 is presented in Fig. 9

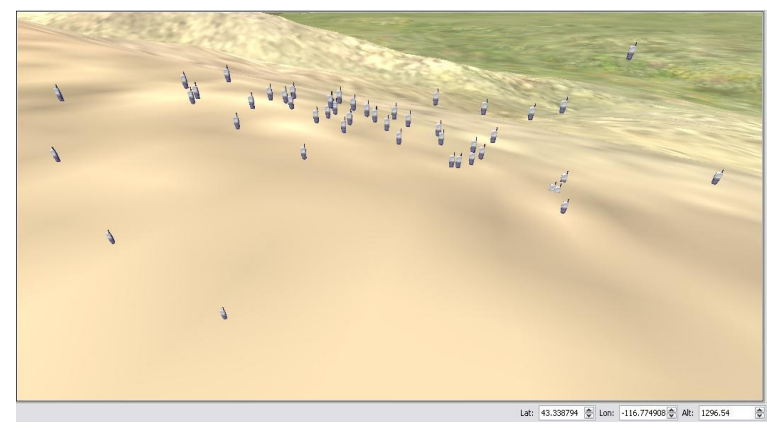

Fig. 9: Topology of the Simulated Network on an Urban Terrain 
The physical medium used is the well known 802.11DSSS PHY with a data rate of 2 mbps. The MAC protocol used is 802.11 MAC protocol, configured in a MANET mode on an urban terrain. More precisely we use only Distributed Coordination Function (DCF) of the protocol.

\subsection{Results}

Table-1 shows parameters we considered for simulations.

Table 1

Parameters we considered for simulations.

\begin{tabular}{|c|c|}
\hline Parameters & Values \\
\hline Routing Protocol & ZRP and Enhanced ZRP \\
\hline Fading Model & Rayleigh \\
\hline Shadowing Model & Constant \\
\hline Pathloss Model & Cost-231 HATA \\
\hline Energy Model & Mica Motes \\
\hline Battery Model & Simple linear \\
\hline Terrain File & DEM \\
\hline Mobility Speed & Area Considered-500 X 500 \\
\hline Network Size-10-20 nodes & Area Considered-1000 X 1000 \\
\hline $\begin{array}{c}\text { Network Size-50-100 } \\
\text { nodes }\end{array}$ & Area Considered-3000 X 3000 \\
\hline Network Size-200 nodes & Random node placement under \\
\hline $\begin{array}{c}\text { Node Placement } \\
\text { seed }\end{array}$ \\
\hline
\end{tabular}

After studying the operation of ZRP and Enhanced ZRP we can observe that, ZRP uses transmission of excess control messages there by resulting in poor QoS, by studying ZRP operation of route discovery we observe that ZRP uses additional time as it uses IARP, IERP and BRP. Thus leads to more number of control packets. When a destination node is not found in the local zone of the source node it initializes IERP. ZRP takes time for inter communication between IERP and IARP. Each node maintains routing table of their local zone. This adds unnecessary traffic in the network. This causes route acquisition delay. After discovering the route to the destination the data packets are encapsulated by two protocols. Hence it takes more time for data packet to reach to the destination.

The average end to end delay introduced is more for ZRP in our test case this is because of flooding more query control messages. Our proposed algorithm reduces this and there by promising reduced End to End delay. This is as shown in below Fig. 10.

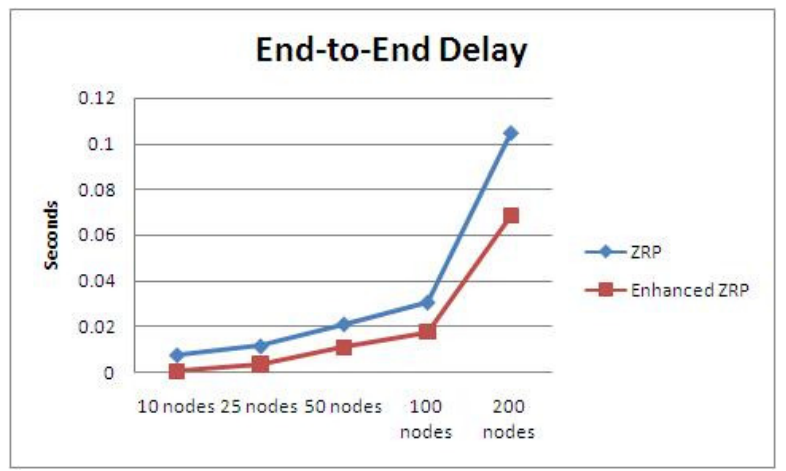

Fig. 10: Average End-to-End delay displayed in seconds for different runs on different network sizes 
The time taken to receive the first packet in case of ZRP is higher when compared to Enhanced ZRP, this is because of the excess control messages flooded in case of IARP and IERP. This is as shown in below Fig. 11.

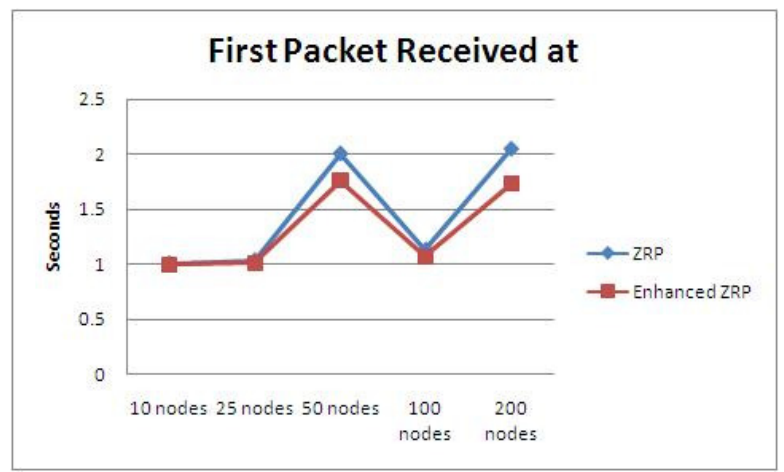

Fig. 11: The first packet received in seconds

In our simulation, time constraint is $100 \mathrm{sec}$, total number of packets and total numbers of bytes at the destination received within this simulation time are reduced for ZRP due to increased delay as compared with Enhanced ZRP. This is as shown in Fig. 12 and Fig. 13 .

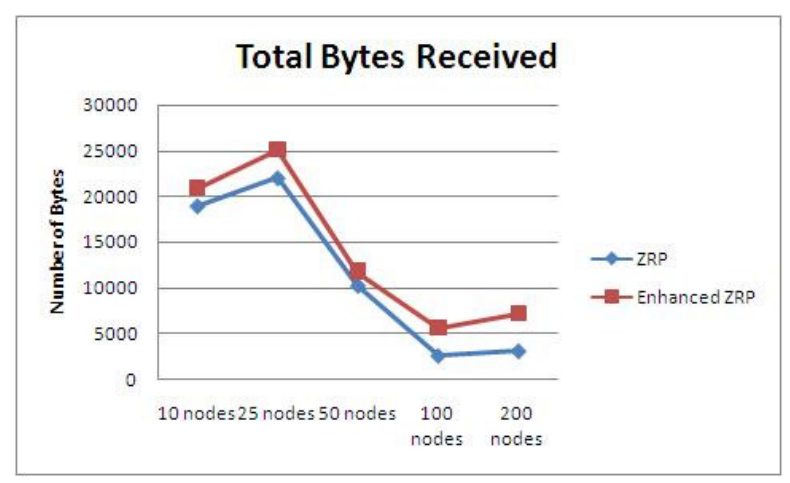

Fig. 12: Total Bytes Received on different network sizes.

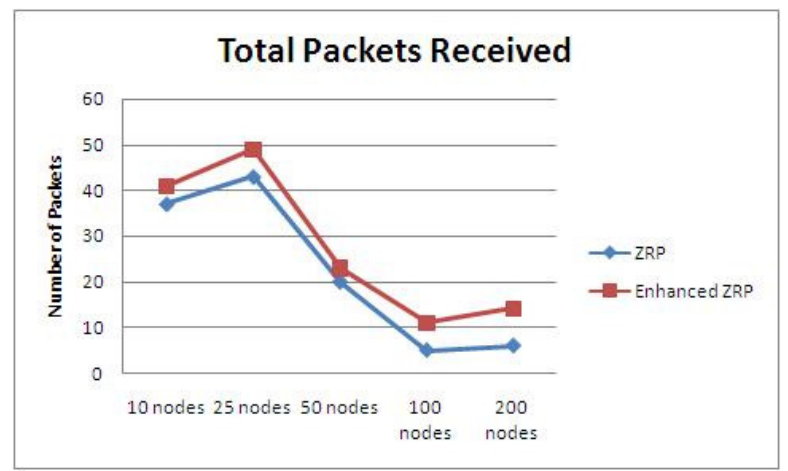

Fig.13: Total packets received for multiple runs with different node density.

In the above Fig. 14 we can observe that when network density is less i.e. for 10 nodes number of packets received are more as compared at high network density. This is because of number of nodes increase, number of overlapping zones increase, thus query messages. 


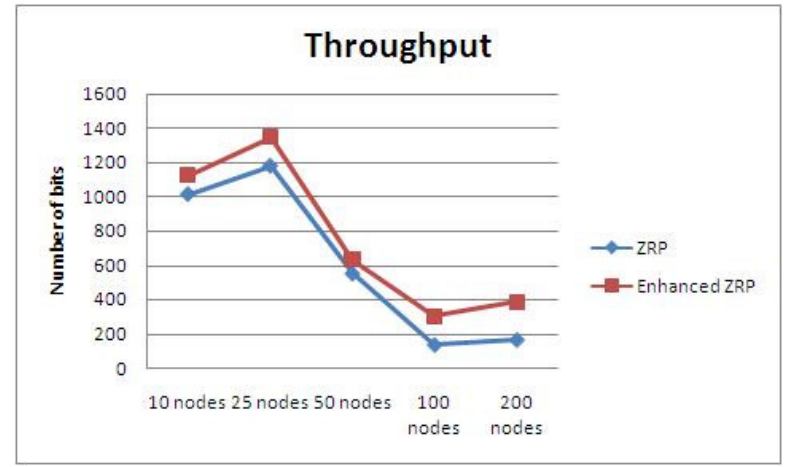

Fig.14: Throughput in bits per second obtained for different network sizes.

As the number of the nodes increases in the network, route discovery becomes more complicated, because centralized node routing zones will highly overlap with each other, hence the route request queries will be flooded in to the network, and the intermediate nodes will send same route request queries multiple times, hence the route acquisition delay will have higher percentage as the number of nodes increases. Therefore the zone routing protocol have lower throughput when compared to Enhanced ZRP. At 200 nodes Enhanced ZRP has low transmission rates but more than ZRP. This is as shown in Fig. 14.

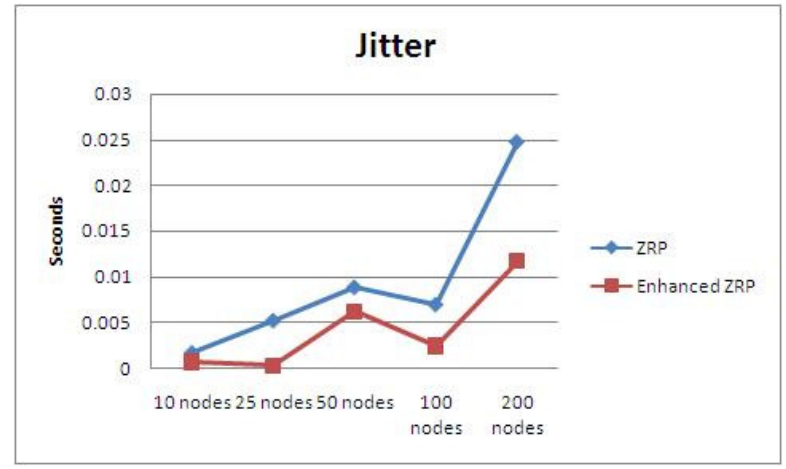

Fig. 15: The average jitter shown in seconds for different network sizes

Jitter is the variation in the time between packets arriving, caused by network congestion, timing drift, or route changes. As from the above Fig. 15 we can observe that at 10 nodes both the protocols have small jitter value. But at 25, 50, 100 and 200 nodes since query packets will be flooded throughout the network control overhead increases; it consumes more time to reconfigure the route if link failure occurs. Hence there will be more time variation between arrivals of packets results in more jitter value.

From above graphs of Fig. 10 thru Fig. 158 we can observe that Enhanced ZRP has performed better than ZRP to promise better throughput, end to end delay, jitter and number of bytes received. ZRP was not up to the task and it performed poorly throughout all the simulation sequences, hence putting itself out of competition. 
International Journal of Wireless \& Mobile Networks (IJWMN) Vol. 3, No. 3, June 2011

\section{Conclusions}

With simulation results we compared ZRP with Enhanced ZRP by involving two enhanced algorithms to enhance the performance of ZRP for communication on MANETs on an Urban Terrain. By analyzing simulation results we can observe Enhanced ZRP had performed better in all the simulation sequences with an improvement of 15 to 20 percent when compared to ZRP. To further improve the efficiency of Enhanced ZRP, our future work shall involve a new algorithm to overcome the generation of periodic updates in the routing table to reduce the control traffic. Thus by the implementation of our proposed algorithm the time taken to receive the first packet will be minimized, which results in better throughput. So the number of bytes and total packets received at the destination will be increased.

The techniques can be applied to single or multiple channel MANETs to improve both the delay and control traffic performance of ZRP. In this scheme, we allow ZRP to provide routes to all accessible network nodes, with less control traffic than purely proactive link state or purely reactive route discovery, and with less delay. Extensive simulation results reveals that our scheme features better transmission delay, route convergence time, the last packet reception time, system efficiency and system through-put.

\section{References}

[1]. Jun-Zhao Sun, "Mobile Ad-Hoc Networking: An Essential Technology for Pervasive Computing," Proceedings of International conference on info-tech and info-net, Vol-3, pp. 316-321, 2001.

[2]. Abdul Hadi Abd Rahman, Zuriati Ahmad Zukarnain, "Performance Comparison of AODV, DSDV and I-DSDV Routing Protocols in Mobile Ad Hoc Networks," European Journal of Scientific Research ISSN 1450-216X, Vol.31 No.4, pp.566-576, 2009.

[3]. P. Jacquet, Ed. Project Hipercom, INRIA "Optimized Link State Routing Protocol (OLSR)," http://www.ietf.org/rfc/rfc3626.txt- DSR-09.txt, IETF Internet draft, Oct. 2003

[4]. Nasipuri and S.R. Das, “On-demand multi-path routing for mobile ad hoc networks," IEEE ICCCN'99, pp. 64-70, 1999.

[5]. S. Singh, M. Woo and C. S. Raghavendra, "Power aware routing in mobile ad hoc networks”, IEEE/ACM MobiCom-98, pp. 181-190. Oct.1998.

[6]. D. Johnson, D. Maltz and Yih-Chun Hu, "The Dynamic Source Routing Protocol for Mobile Ad Hoc Networks," http://www.ietf.org/internet- drafts/draftietfmanet- DSR-09.txt, IETF Internet draft, Apr. 2003.

[7]. Vincent D. Park and M. Scott Corson, "Adhoc On demand Distance Vector (AODV) version 4: Functional specification,” Internet- Draft,draft-ietfmanet- AODV-spec-04.txt, July 2001.

[8]. Zygmunt J. Haas, Marc R. Pearlman,Prince Samar, “ The Zone Routing Protocol (ZRP) for Ad Hoc Networks,” draft-ietf-manet-zone-zrp-04.txt, July 2002.

[9]. Zygmunt J. Haas, Marc R. Pearlman,Prince Samar, “The Intrazone Routing Protocol (IARP) for Ad Hoc Networks," draft-ietf-manet-zone- iarp-01.txt, June 2001.

[10]. Zygmunt J. Haas, Marc R. Pearlman,Prince Samar, "The Interzone Routing Protocol (IERP) for Ad Hoc Networks,” draft-ietf-manet-zone- ierp 02.txt July 2002.

[11]. C. Perkins, E.M. Royer, S.R. Das, and M.K. Marina, "Performance Comparison of Two Ondemand Routing Protocols for Ad Hoc Networks,” IEEE Personal Communications, pp. 1628, Feb. 2001

[12]. J. Broch et al., "A Performance Comparison of Multihop Wireless Ad Hoc Network Routing Protocols,” Proc. IEEE/ACM MOBICOM '98, pp. 85-97, Oct. 1998. 
International Journal of Wireless \& Mobile Networks (IJWMN) Vol. 3, No. 3, June 2011

[13]. Pearlman MR, Haas ZJ, "Determining the optimal configuration for the zone routing protocol," IEEE Journal on Selected Areas in Communications 1999, vol. 17, No.8, pp. 1395-1414, AUGUST 1999.

[14]. Zygmunt J. Haas, and Marc R. Pearlman, "The Performance of Query Control Schemes for the Zone Routing Protocol” IEEE/ACM TRANSACTIONS ON NETWORKING, VOL. 9, NO. 4, pp. 427-438, AUGUST 2001.

[15]. Idris Skloul Ibrahim A. Etorban Dr Peter J.B King, "Multipath Distance Vector Zone Routing Protocol for Mobile Ad-Hoc Networks MDVZRP," PGnet 2008, John Moores University, pages 171-176, July 2008.

[16]. Leonard Barolli, Yoshitaka Honma , Akio Koya, Arjan Durresi and Junpei Arai, “ $A$ Selective Border-casting Zone Routing Protocol for Ad-hoc Networks," Proceedings of the 15th International Workshop on Database and Expert Systems Applications (DEXA'04), pp. 326330,2

[17]. Kavita Taneja and R.B.Patel," Mobile Ad hoc Networks: Challenges and Future", Proceedings National Conference on Challenges and Opportu-nities in Information Technology, RIMT-IET, Mandi Gobindgarh, PP.133-135, March 3, 2007

[18]. Prince Samar, Marc R. Pearlman and Zygmunt J. Haas,Independent Zone Routing:An Adaptive Hybrid Routing FrameWork for Ad hoc Wireless Networks",IEEE/ACM Transaction On Networking,Vol.12, No. 4, PP. 595-608, August 2004.

[19]. Moitreyee Dasgupta, S. Choudhury and N. Chaki," Routing Misbehavior in Ad Hoc Network", International Journal of Computer Applications, Vol.1, No. 18, PP.89-92, 2010

[20]. Ming Yu and Kin K. Leung, "A trustworthiness-based QoS routing protocol for wireless ad hoc networks", IEEE Transactions on Wireless Communications, Vol. 8, No. 4, April 2009

[21]. P. Papadimitratos and Zygmunt J. Haas, Secure Message Transmission in Mobile Ad hoc Networks",Journal Elsevier Ad hoc Networks, Vol. 1, No.1 PP. 193-209, 2003.

Dr. Jithendranath Mungara received B.S.E.E degree from U.S.A, M.Tech and Ph.D from Andhra University, AP, India.

He has teaching and research experience more than a decade. He has served as Principal, in BTLIT, Bangalore and currently working as Director and Dean PG studies and research at CMRIT, Bangalore, India. His research interests includes MANETS, Protocol design, Computer Networks, Software Engineering and cloud computing.

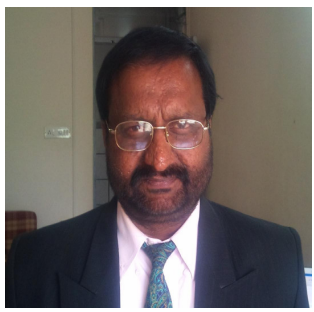
Dr. Jithendranath has organised several national and international conferences and workshops, delivered numerous tutorials at major conferences held in India and Abroad

Prof. M.N. Sree Ranga Raju received B.E (Bangalore University) in 1985 , M.Tech (Mysore University) in 1991 and currently a Ph.D Candidate in Telecommunication Engg, at VMU, Salem, Tamilnadu, India.

He has teaching experience more than a two and half decade. He is currently Professor and Head, Department of Telecommunication Engg, BIT, Bangalore, India.

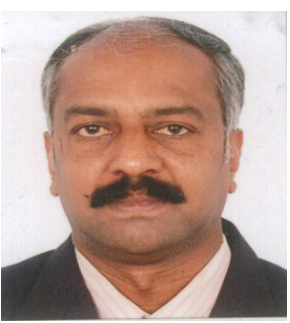

His research interest includes routing protocol design for MANETS and Wireless Channel Modelling for Adhoc Network Simulation.

M.N. Sree Ranga Raju has organised several national conferences and workshops. 